\title{
Structure and Function of Bacterial Kid-Kis and Related Toxin-Antitoxin Systems
}

\author{
Monique B. Kamphuis ${ }^{1}$, Maria Chiara Monti ${ }^{2, \$}$, Robert H. H. van den Heuvel $^{2}$, \\ Juan López-Villarejo ${ }^{3}$, Ramón Díaz-Orejas ${ }^{3}$ and Rolf Boelens ${ }^{1, *}$
}

\begin{abstract}
${ }^{I}$ Bijvoet Center for Biomolecular Research, Department of NMR Spectroscopy, Utrecht University, Padualaan 8, 3584 CH Utrecht, The Netherlands; ${ }^{2}$ Bijvoet Center for Biomolecular Research, Department of Biomolecular Mass Spectrometry, Utrecht University, Sorbonnelaan 16, 3584 CA Utrecht, The Netherlands; ${ }^{3}$ Centro de Investigaciones Biológicas, Departamento de Microbiología Molecular, Ramiro de Maeztu 9, E-28040 Madrid, Spain
\end{abstract}

\begin{abstract}
Toxin-antitoxin systems were discovered as plasmid auxiliary maintenance cassettes. In recent years, an increasing amount of structural and functional information has become available about the proteins involved, allowing the understanding of bacterial cell growth inhibition by the toxins on a molecular level. A well-studied TA system is formed by the proteins Kid and Kis, encoded by the parD operon of the Escherichia coli plasmid R1. The toxicity of Kid has been related to its endoribonuclease activity, which is counteracted by binding of the antitoxin Kis at the proposed active site. In this review, the structural studies on the Kid-Kis system are compared to those of three related toxin-antitoxin systems: MazF-MazE, CcdB-CcdA and RelE-RelB.
\end{abstract}

Keywords: CcdB-CcdA, MazF-MazE, plasmid maintenance, RelE-RelB, ribonuclease.

\section{TOXIN-ANTITOXIN SYSTEMS}

During bacterial growth, replication of the chromosome and plasmids takes place prior to cell division. Although plasmids are not crucial for the proliferation of bacteria, the valuable genetic information they carry can be of great benefit for the bacterial cell line in particular circumstances. Antibiotic resistance factors confer to their bacterial hosts the capacity to grow in the presence of antibiotics, and catabolic plasmids in Pseudomonas increase the nutritional versatility of the cells by allowing the utilisation of new substances, for instance aromatic compounds, as source of carbon and energy. In spite of the non-essential character of plasmids, these extrachromosomal genetic elements are maintained stably in the cells by a variety of auxiliary stability systems. Some of these systems prevent the growth of plasmid-free progeny via a programmed cell death mechanism called post-segregational killing (PSK) [1-4].

\section{PSK-Inducing Plasmids}

Two different types of PSK-inducing plasmids are known that function in a highly similar way. They contain genes that encode a stable protein toxin and an unstable antitoxin that can either be an antisense ribonucleic acid (RNA) or a protein antitoxin [2,5]. The antitoxin inhibits the activity of the toxin. However, plasmid loss during cell division is lethal, since the unstable antitoxin cannot be replenished upon degradation $[3,4,6]$. In the antisense RNA-regulated killing sys-

*Address correspondence to this author at the Department of NMR Spectroscopy, Utrecht University, Padualaan 8, $3584 \mathrm{CH}$ Utrecht, The Netherlands; Tel: +31 30253 4035/2652; Fax: +31 30253 7623; E-mail: r.boelens@chem.uu.nl

${ }^{\ddagger}$ Present address: Dipartimento di Scienze Farmaceutiche, Universita' di Salerno, Via Ponte Don Mellilo, 84084, Fisciano, Salerno, Italy tems, the toxin is only synthesised when the antitoxin is absent, because only then translation of the inherited toxinencoding messenger RNAs (mRNAs) can take place. Subsequently, the produced toxins cause bacterial death by damaging the bacterial cell membrane $[4,7]$. The protein antitoxinregulated systems are called plasmid addiction modules or more generally toxin-antitoxin (TA) systems. In these systems, the toxin is already present during normal growth of bacteria. The antitoxin neutralises the toxicity by forming a protein complex with the toxin. Upon loss of the plasmid and therefore a diminished level of antitoxin, the free toxin will exert its lethal potential $[3,5,6,8]$.

\section{TA Systems Are Also Present on Chromosomes}

Originally, TA systems were discovered in plasmids, but they are also highly abundant in bacterial as well as archaeal genomes [6,9]. In fact, a large conservation exists between several plasmid- and chromosome-encoded systems. As already described for plasmid-encoded TA systems, also chromosomal TA systems are involved in cell growth arrest. However, for the latter systems the toxin activity does not necessarily result in cell death; the expression of toxicity can be reversed since new antitoxin can be synthesised [6,10-14]. Several systems are thought to function in quality control of gene expression and to provide a control mechanism for freeliving prokaryotes to cope with nutritional stress $[13,15,16]$. Also, a relation between bacterial TA systems and the eukaryotic nonsense-mediated RNA decay system has been suggested $[9,17]$.

\section{Applications of TA Systems}

Interest in bacterial toxins is given by their potential use in the development of new antibiotics $[6,10,15]$. These antibiotics could mimic the lethal action of the toxins or trigger 
the activation of toxins already present in bacterial cells. The latter option appears very promising since many prokaryotes carry multiple TA loci, including pathogens such as Mycobacterium tuberculosis and Vibrio cholerae. Furthermore, several bacterial TA systems were reported to function in yeast and induce apoptosis in human cells [18-20]. Possibly, these toxins can be converted into medicines that can prevent uncontrolled division of malignant cells in humans. Another valuable application of TA systems is their use in biotechnical selection vectors. Like many antibiotics, the toxins are used to achieve high selective efficiency of recombinants [21-23].

\section{Five of the Best-Known TA Systems}

In an exhaustive and recent search for TA loci in 126 completely sequenced prokaryotic genomes, 671 loci were found that could be grouped in seven known TA families [15]. These are, in order of discovery of the plasmid-encoded systems: the ccd locus of plasmid F [24], the parD (pem) locus of R1/R100 [25], the vapBC locus of the Salmonella Dublin virulence plasmid [26], the phd/doc locus of plasmid $\mathrm{P} 1$ [27], the parDE locus of plasmid RK2 [28], the higBA locus of plasmid Rts1 [29], and the relBE locus of plasmid P307 [30]. The chromosomal TA systems mazEF and $c h p B$ are members of the $\mathrm{parD/pem}$ family [31,32]. MazEF, identified being a TA system by the group of Ohtsubo, was initially called chpA which refers to chromosomal homologue of pem [32]. Chromosome-encoded relBE, yefM-yoeB and dinJ-yafQ are chromosomal homologous of the plasmidencoded relBE system and therefore members of this family $[9,16,33]$. However, it has been discussed that yefM-yoeB and $\mathrm{relBE}$ may belong to different families as their toxins and antitoxins differ substantially either functionally or structurally (Cherny and Gazit, 2004). The RelE, ParE and HighB toxins form a superfamily of toxins [9], and the $\mathrm{Kid} / \mathrm{PemK}$ and CcdB toxins form another superfamily [34]. An orphan family of the TA systems that is unrelated to the seven previously described families was identified in plasmid pSM19035 of Streptococcus pyogenes and comprises the toxin $\zeta$, the antitoxin $\varepsilon$ and a third component, the $\omega$ regulator $[35,36]$.

This review describes five related TA systems, all found in E. coli: the plasmid-encoded Kid-Kis system, its chromosomal homologue the MazF-MazE system, the CcdB-CcdA system, the RelE-RelB system, and finally the YoeB-YefM system. In recent years, the biochemical knowledge about these TA systems has increased enormously and structural information about all five toxins and antitoxins has become available [34,37-43]. Fig. 1 contains the 3D structures of the toxins $\mathrm{Kid}$ and $\mathrm{CcdB}$ shown in their unbound state, while RelE, YoeB, and MazF are depicted in complex with their antitoxins RelB, YefM, and MazE, respectively.

\section{THE Kid-Kis SYSTEM}

The toxin Kid (Killing determinant; $11.9 \mathrm{kD}$ ) and antitoxin Kis (Killing suppressor; $9.5 \mathrm{kD}$ ) are encoded by the parD locus of the E. coli plasmid R1 [25]. The proteins are identical to PemK and PemI, respectively, of the pem (plasmid emergency maintenance) locus of plasmid R100 [44]. Another parD-homologous locus has been identified on plasmid R466B of Morganella morganii [16]. The sequence of this toxin is almost identical to that of Kid, since only four out of 110 amino acids are different: V64A, N99D, E100D, and S106A.

\section{Common Phenomena}

Kid has been demonstrated to inhibit cell growth in $E$. coli and Kis counteracts this effect [45]. The coordinate action of Kid and Kis has been shown to autoregulate parD at the level of transcription [46-49]. This kind of transcriptional control is generally observed for TA systems. The antitoxin represses transcription $[33,47,49-51]$ by binding the operator with the N-terminus [48,52,53]. The toxin acts as a corepressor, since the TA complex has a higher DNA-affinity than the antitoxin alone $[33,46,53,54]$. Another phenomenon common for TA systems is the constant degradation of the unstable antitoxin by proteases. The half-lives of antitoxins are in the range of 15 to 60 minutes in vivo [55,56]. A mutant of the Lon protease prevents the activation of the toxin Kid, which indicates that Kis is degraded by the native Lon protein [57].

\section{Structures and Interactions}

The crystal structure of the Kid protein reveals a two-fold symmetric dimer, as shown in Fig. 1. Each spherical monomer consists of a five-stranded twisted antiparallel $\beta$-sheet and a 12 residue $C$-terminal $\alpha$-helix with a small threestranded antiparallel $\beta$-sheet inserted in the main sheet, two additional $\alpha$-helices of seven and three residues, and two loop regions [34,58]. A salt-bridge was observed between R85 and E18, linking the two Kid monomers [59]. The secondary structure elements of both Kid and Kis in solution were determined using NMR spectroscopy (Fig. 2) [43]. A high structural similarity exists between the crystal and solution states of Kid, between Kid and MazF and between Kis and MazE (Fig. 2). The interactions between Kid and Kis that are responsible for neutralisation of Kid toxicity and enhance autoregulation of parD transcription have been investigated [43]. Native macromolecular mass spectrometry data have demonstrated that Kid and Kis can form multiple complexes, depending on their precise molar ratios. At Kis:Kid ratios equal to or exceeding $1: 1$, as found in vivo in a plasmid-containing cell, various complexes are present: from $\mathrm{Kid}_{2}-\mathrm{Kis}_{2}$ tetramer up to $\mathrm{Kis}_{2}-\mathrm{Kid}_{2}-\mathrm{Kis}_{2}-\mathrm{Kid}_{2}-\mathrm{Kis}_{2}$ decamer. When Kid is in excess of Kis, corresponding to an in vivo situation immediately after loss of the plasmid, the $67 \mathrm{kD}$ $\mathrm{Kid}_{2}-\mathrm{Kis}_{2}-\mathrm{Kid}_{2}$ heterohexamer is the most abundant species. NMR titration experiments showed that the interaction sites of $\mathrm{Kid}$ and $\mathrm{Kis}$ resemble those within the $\mathrm{MazF}_{2}-\mathrm{MazE}_{2}-$ $\mathrm{MazF}_{2}$ complex, which will be described later [38,43]. Fig. 3 shows the Kid-Kis interactions mapped on a MazF-MazE based model and the crystal structure of the Kid dimer. It was reported that the affected Kid residues K83 and A84, located in the small $\alpha$-helix within interaction site 1 , might alternatively be part of the other Kid monomer compared to the situation shown here [60]. Overproduction of MazE has been demonstrated to neutralise Kid toxicity to a certain extent in the absence of native Kis. It was shown that $\mathrm{Kid}_{2}-$ $\mathrm{MazE}_{2}$ tetramers can be formed via weak interactions involving a limited part of the Kis-binding residues of Kid [43]. The results imply that interactions between $\mathrm{Kid}$ and the $\mathrm{N}$ - 

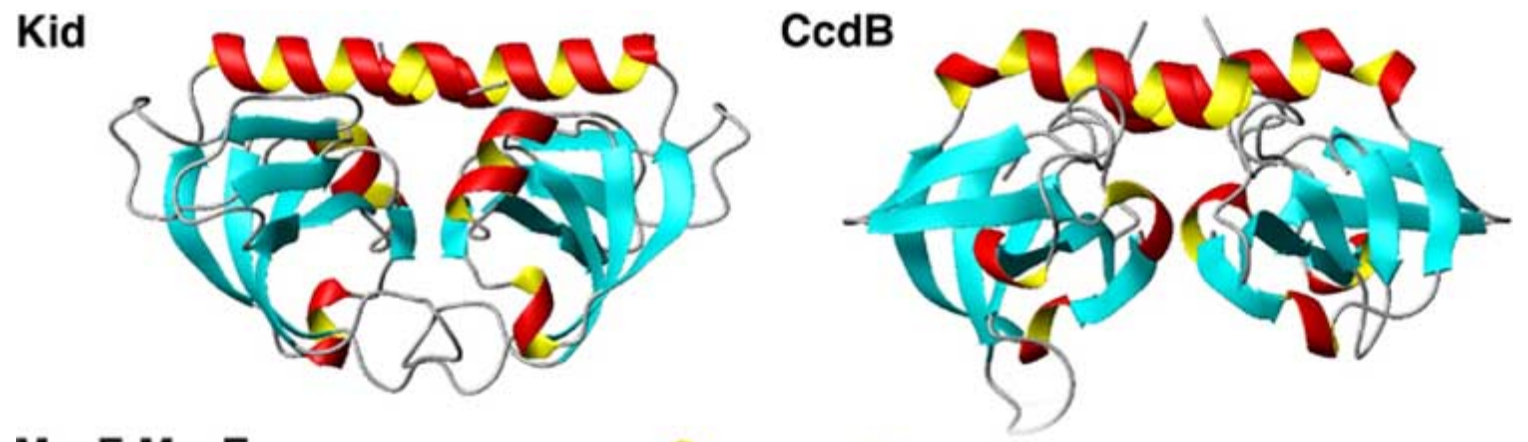

MazF-MazE

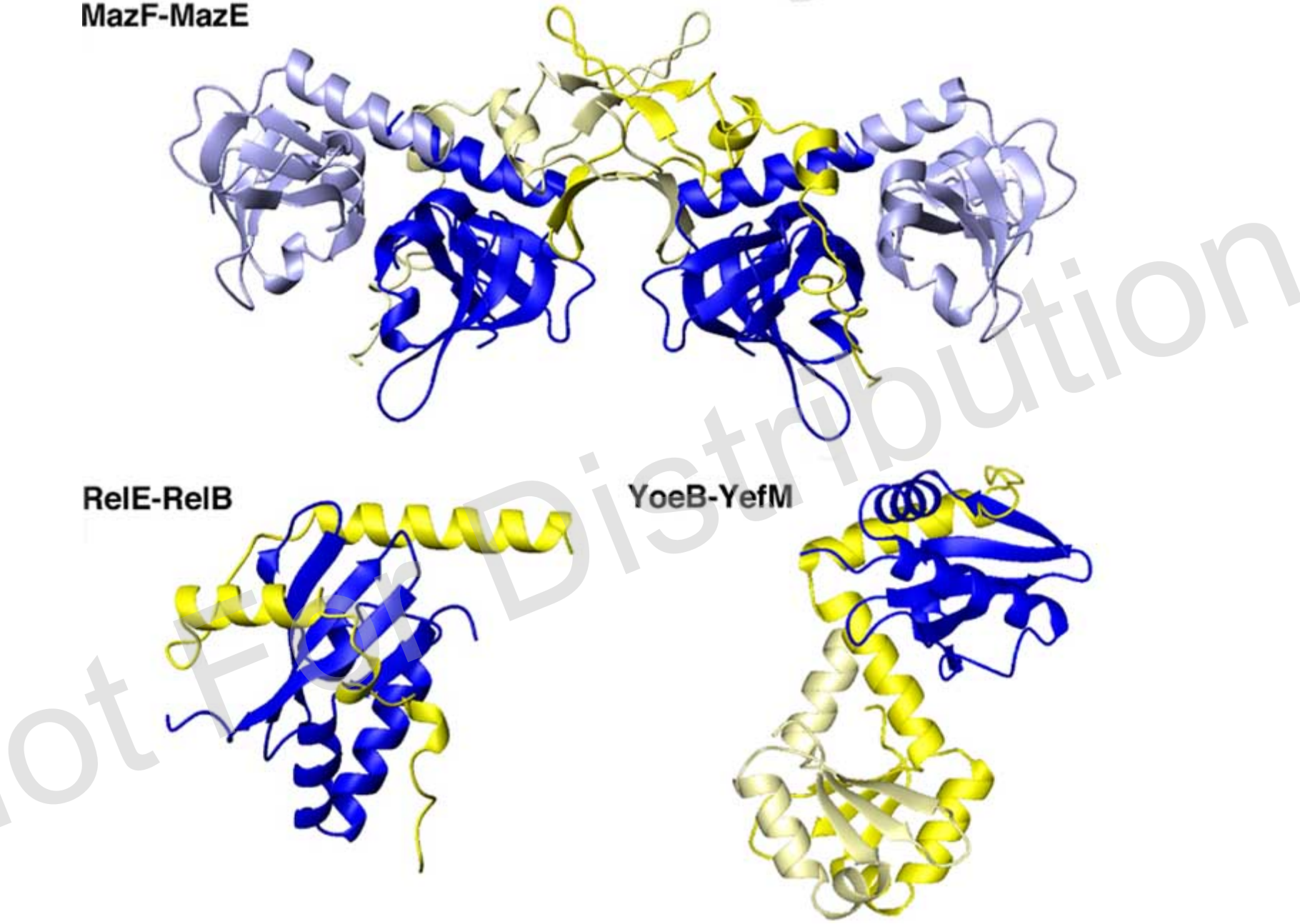

20

Figure 1. Ribbon representations of the crystal structures of the toxins Kid (PDB: 1M1F) and CcdB (PDB: 3VUB) and the toxin-antitoxin complexes MazF-MazE (PDB:1UB4), aRelE-aRelB (PDB: 1WMI) and YoeB-YefM (PDB: 2A6Q). The dimeric Kid and CcdB proteins are shown with their secondary structure elements highlighted ( $\alpha$-helices coloured red/yellow and $\beta$-strands cyan). For the heterohexameric $\mathrm{MazF}_{2}-\mathrm{MazE}_{2}-\mathrm{MazF}_{2}$ complex, the heterodimeric RelE-RelB complex, and the heterotrimeric YoeB-YefM complex, the toxin monomers are coloured dark/light blue and the antitoxin monomers dark/light yellow. The figures were generated with the program MOLMOL version $2 \mathrm{~K}$. 1 [109].

terminal part of Kis, known to be involved in autoregulation [48], likely induce a proper antitoxin-to-toxin orientation and antitoxin monomer-monomer stabilisation, which both seem to be important for effective transcriptional repression of the parD operon.

\section{Mode of Action of the Kid Toxin}

Kid has been reported to inhibit protein synthesis in cell extracts, and recently it was demonstrated that in vitro, the toxin acts as an endoribonuclease able to cleave RNA in the absence of ribosomes. Kid cleaves RNA preferentially at the 5' side of the A residue in the nucleotide sequence 5'$\mathrm{UA}(\mathrm{A} / \mathrm{C})-3^{\prime}$ of single-stranded regions, although cleavage at the 3' side of the adenosine has been observed as well $[61,62]$. Recent experiments by De la Cueva-Méndez and coworkers have indicated that the 5'-UUACU-3' sequence is a longer, more specific target of Kid [63]. Cleavage of the dinucleotide UpA and the 5-nucleotide RNA fragment 
a

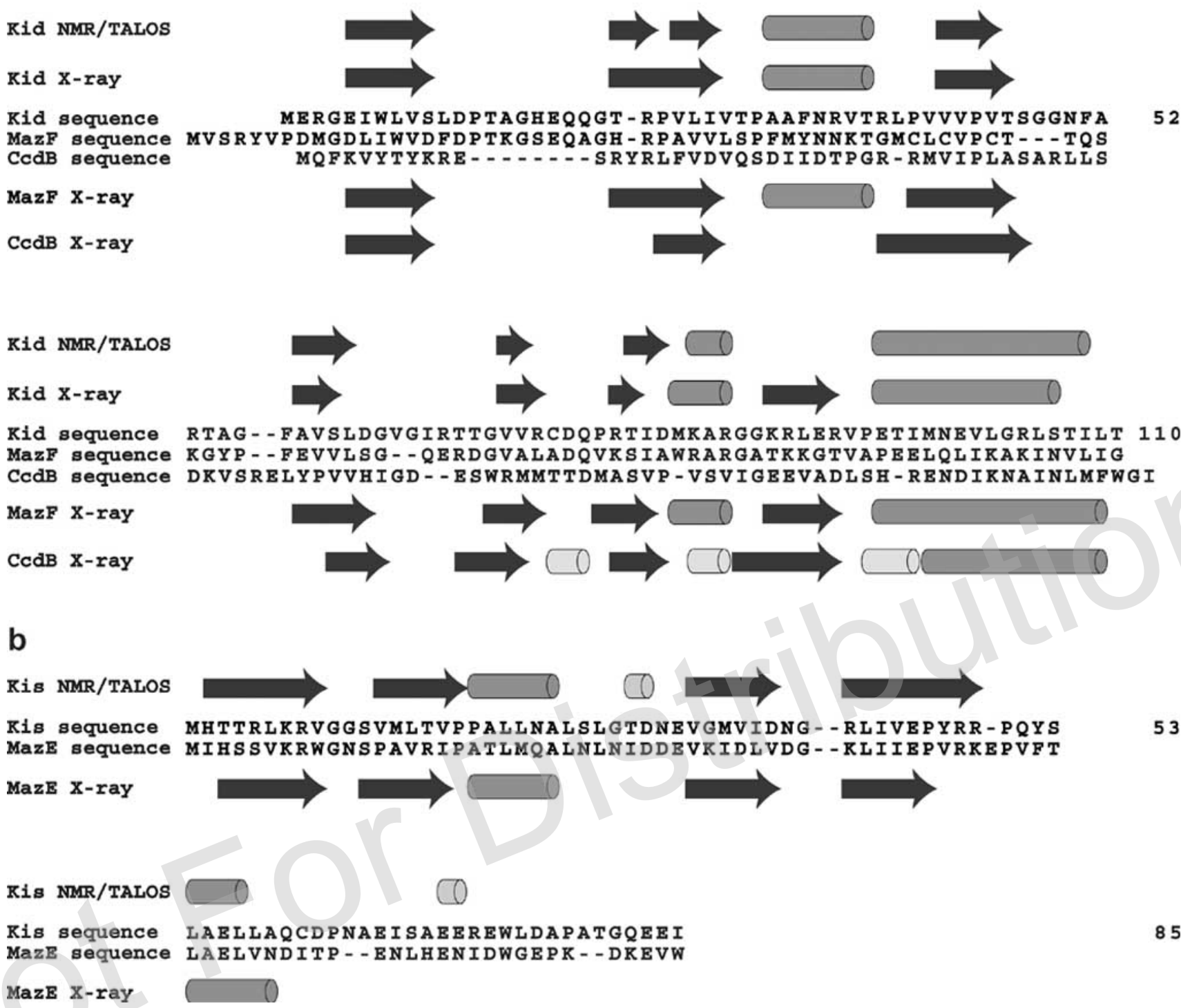

Figure 2. Sequence alignments and secondary structure elements of (a) the structurally related toxins Kid, MazF and CcdB (PDB: 1M1F, $1 \mathrm{UB} 4$ and 3VUB), and (b) the structurally related antitoxins Kis and MazE (PDB: n.a. and 1UB4). $\alpha$-helices are shown as light grey cylinders and $\beta$-sheets as dark grey arrows. Very light grey cylinders represent $3{ }_{10}$-helices for CcdB and helical turns for Kis. The method used to determine the secondary structure, i.e. NMR, NMR/TALOS or X-ray spectroscopy, is indicated. The sequence alignments are shown according to Kamada et al. (2003).

AUACA yields two products with an uracyl 2':3'-cyclic phosphate at one side and an adenosine with a free 5'-OH group at the other side [60]. The uracyl 2'-OH group is essential for cleavage [60], like in the cleavage mechanisms of the rather distinct RNases A and T1. RNA cleavage by these two ribonucleases is well-studied and illustrated by Fig. 4: In a transphosphorylation reaction the 2'-hydroxyl group is deprotonated by a catalytic base to perform a nucleophilic attack on the electrophilic phosphorus. A catalytic acid donates a hydrogen atom to the 5'-leaving group. In the readily reversible second step, a 3'-monophosphate nucleotide is formed by hydrolysis of the 2':3'-cyclophosphate [64-67]. Inouye and co-workers concluded that Kid cleaves only single-stranded cellular mRNAs [62]. However, Díaz-Orejas and co-workers reported that also efficient cleavage of some target sequences located in double-stranded RNA regions and primer RNA takes place, and that Kid likely inhibits RNA-regulated cellular processes in general [61]. It was shown that Kid becomes active in plasmid-containing cells upon the decrease of the R1 copy number, instead of only in plasmid-free cells as originally thought, and that in this situation the R1 plasmid copy number is restored [68]. More recently it was found that cleavage by Kid of a $5^{\prime}$-UUACU-3 sequence located close and $3^{\prime}$ to the CopB mRNA region can lead to decreased levels of the CopB mRNA and therefore of the CopB protein that limits initiation of $\mathrm{R} 1$ replication, thus favouring increased replication of this plasmid [63].

\section{RNA Binding and Cleavage by Kid}

The mechanism of RNA binding and cleavage by the Kid toxin has been determined in considerable detail via NMR titration studies with the uncleavable single-stranded RNA 
mimic AdUACA [60]. It was demonstrated that residues of both monomers of the Kid dimer are needed to form a RNA binding surface. These residues cluster into five sequencebased groups: cluster \#1 contains residues 10-23, cluster \#2 residues 36-41, cluster \#3 residues 47-59, cluster \#4 residues 69-76 and cluster \#5 residues 82 and 83. Clusters \#1, \#3 and \#4 of monomer A cover a concatenated surface area together with clusters \#2 and \#5 of monomer B, forming the first RNA binding site, and vice versa for the second binding site. This implies that Kid is toxic for bacterial cells only in its dimeric form, in contrast to RNase $\mathrm{T} 1$ and RNase A. The stoichiometry of the $\mathrm{Kid}_{2}$-RNA complex was established to be $1: 1$, although Kid is a symmetric dimer. Comparison of the RNA and Kis binding sites of Kid (Fig. 3) clearly points out, that upon binding of the antitoxin, one of the RNA binding sites will be partly occupied by the C-terminal tail of the protein [43]. This result is in agreement with mutagenesis data showing the antidote function of this part of Kis [48]. The associated "opening" of the loop between $\beta$-strands 1 and 2 will disrupt the second RNA binding pocket of Kid [60], thus explaining the way in which Kis prevents RNA cleavage by Kid $[61,62]$. A detailed picture of the position of the 5-nucleotide RNA fragment AUACA within the nucleotide binding pocket of Kid (Fig. 3) has been obtained via docking calculations performed with the program HADDOCK $[60,69]$. Those calculations were based on the NMR chemical shifts, the cleavage reaction and mutagenesis data showing that two clusters of residues of Kid (V9/E18/G21/V25 and G70/R73/D75/D81/R85/P94) are important for the toxicity of the protein $[59,60]$. It was proposed that residues D75, R73 and $\mathrm{H} 17$ form the active site of the Kid toxin (Fig. 4). With help of R73, the catalytic base D75 deprotonates the 2'-OH group of uracyl, which subsequently performs a nucleophilic attack on the electrophilic phosphorus. The transphosphorylation reaction is complete after donation of a hydrogen atom by the catalytic acid R73 to the adenosine 5'-O. H17 stabilises the complex, and the RNA sequence specificity is defined by direct interactions of Kid residues T46, S47, A55, F57, T69, V71 and R73 with the RNA nucleotides UA(A/C) [60].

\section{Kid-Ligand Interactions}

The interactions of Kid with the potential inhibitors 5'AMP, 5'-UMP, 5'-CMP, 5'-GMP, 2':3'-cUMP, Pi, the minimal substrate $\mathrm{UpA}$, and $\mathrm{Mg}^{2+}$ were studied using NMR spectroscopy and mass spectrometry. The RNA cleavage product 2':3'-cUMP was found to be the most potent Kid inhibitor among those ligands [70]. In the presence of 0.5 mM 2':3'-cUMP, Kid cleaves the RNA oligonucleotide with a catalytic efficiency of $0.60 \mathrm{M}^{-1} \mathrm{~s}^{-1}$ instead of $0.78 \mathrm{M}^{-1} \mathrm{~s}^{-1}$. If any inhibition takes place by the other ligands, this effect is very weak and does not result in a significant decrease of the Kid activity. The Kid residues involved in protein-ligand interactions were identified via NMR titration experiments [70]. Addition of 2':3'-cUMP resulted in the largest chemical shift changes, showing a nice correlation between the size of the NMR chemical shift perturbations and the inhibitory effect of the compounds. The addition of any of the five mononucleotides resulted in a chemical shift perturbations pattern that is, although weaker, almost identical to the effect of AdUACA binding (Fig. 5). All Kid residues suggested being involved in interactions with the target sequence UAC (H17, R23, T37, T46, S47, A55, F57, T69, V71, R73 and D75) were observed in this Kid-inhibitor interaction study. Interestingly, the catalytic acid R73 only shows a perturbation upon addition of $2^{\prime}: 3^{\prime}$-cUMP, and the catalytic base D75 only when 2':3'-cUMP or 5'-CMP is added. Clearly, the cleavage product 2':3'-cUMP shows the strongest interactions with the RNA binding pocket and catalytic residues of Kid. Since 2':3'-cUMP also inhibits the endoribonuclease activity of the toxin, it appears to be the most promising lead for designing an effective regulator of Kid activity in both prokaryotic and eukaryotic cells.

\section{THE MazF-MazE SYSTEM}

The toxin MazF (12.0 kD) and antitoxin MazE (9.3 kD) are chromosomal homologues of Kid and Kis, respectively. They are encoded by the E. coli mazEF locus ('ma-ze' is Hebrew for 'what is it?') and are also known as ChpAK respectively ChpAI [6,31,32]. Sixty-six other chromosomal $m a z E F$ loci have been identified in both gram-negative and gram-positive organisms. Publications include the characterisation of homologous TA systems in Bacillus subtilis [71,72], Streptococcus mutans [73] and M. tuberculosis [74].

\section{Structures and Interactions}

The structure of the complex of MazF and MazE is shown in Fig. 1. In this structure, the two proteins form a linear heterohexamer made up by alternating toxin and antitoxin homodimers $\left(\mathrm{MazF}_{2}-\mathrm{MazE}_{2}-\mathrm{MazF}_{2}\right)$ [38]. The overall structure of MazF resembles that of its plasmid-encoded homologue, Kid. The MazE homodimer contains an Nterminal intertwined $\beta$-barrel and two mainly unfolded $\mathrm{C}$ terminal tails that are each bound to a MazF dimer interface, thereby mediating the main interactions between MazE and MazF [38,39]. The TA complex has been shown to autoregulate $m a z E F$ expression via binding of the DNA by the Nterminal domain of MazE [50,53]. During steady state, the antitoxin is degraded by the protease ClpAP (caseinolytic protease AP) [75], while during amino acid starvation Londependent activation of transcription of $m a z E F$ was observed [76].

\section{Mode of Action of the MazF Toxin}

It was demonstrated that MazF cleaves translated mRNAs and blocks protein synthesis, a blockage which is counteracted by tmRNA [76]. Like Kid, MazF has been shown to inhibit protein synthesis in cell extracts and act as an endoribonuclease able to cleave RNA in the absence of ribosomes in vitro [77-79]. This toxin was reported either to cleave XACA sequences at the 5' end of ACA yielding a $2 ': 3^{\prime}$-cyclic phosphate at one side and a free 5'-OH group at the other side [77,78], or to cleave at the 5' end of residue A in NAC sites (where $\mathrm{N}$ is $\mathrm{U}$ or $\mathrm{A}$ ) [79]. The 2'-hydroxyl group of de nucleotide preceding the adenosine was shown to be essential for RNA cleavage [78]. As for Kid, the precise nature of the MazF substrate is subject to discussion. It could be only single-stranded mRNAs [77,78], or alternatively, all cellular RNAs including both single- and doublestranded RNA [79]. Ikura and co-workers used a singlestranded DNA fragment in a NMR titration experiment to 

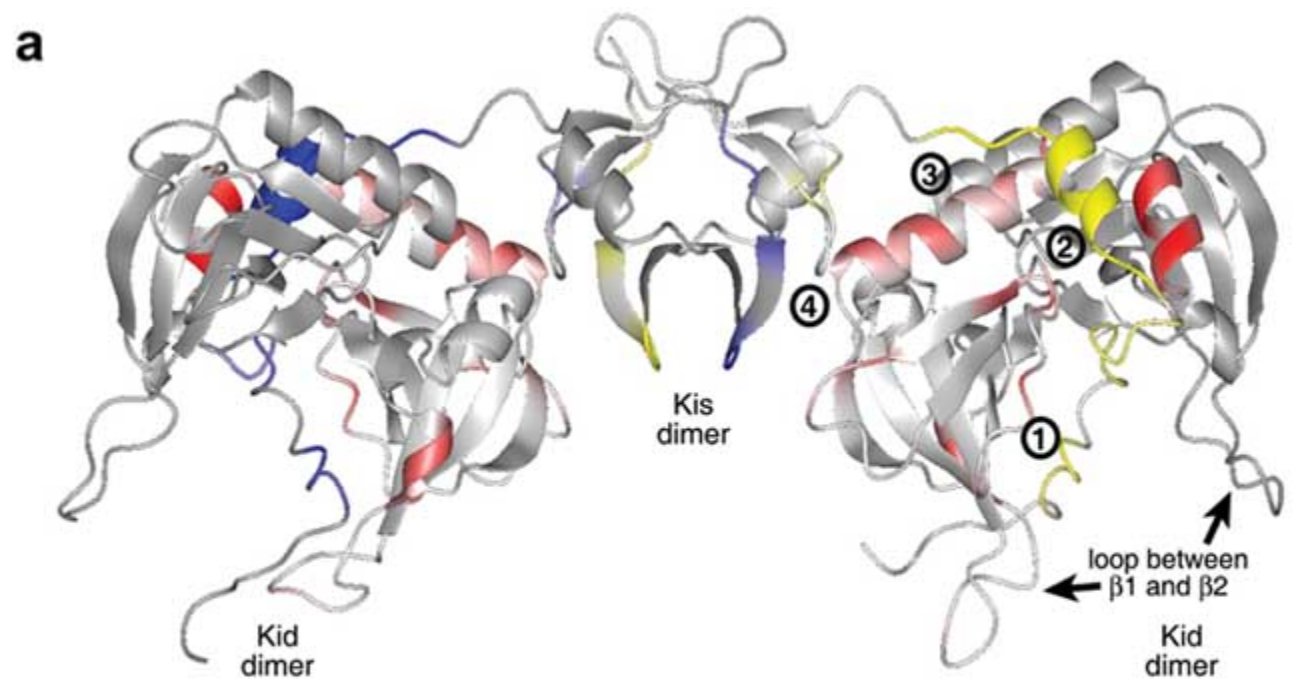

b
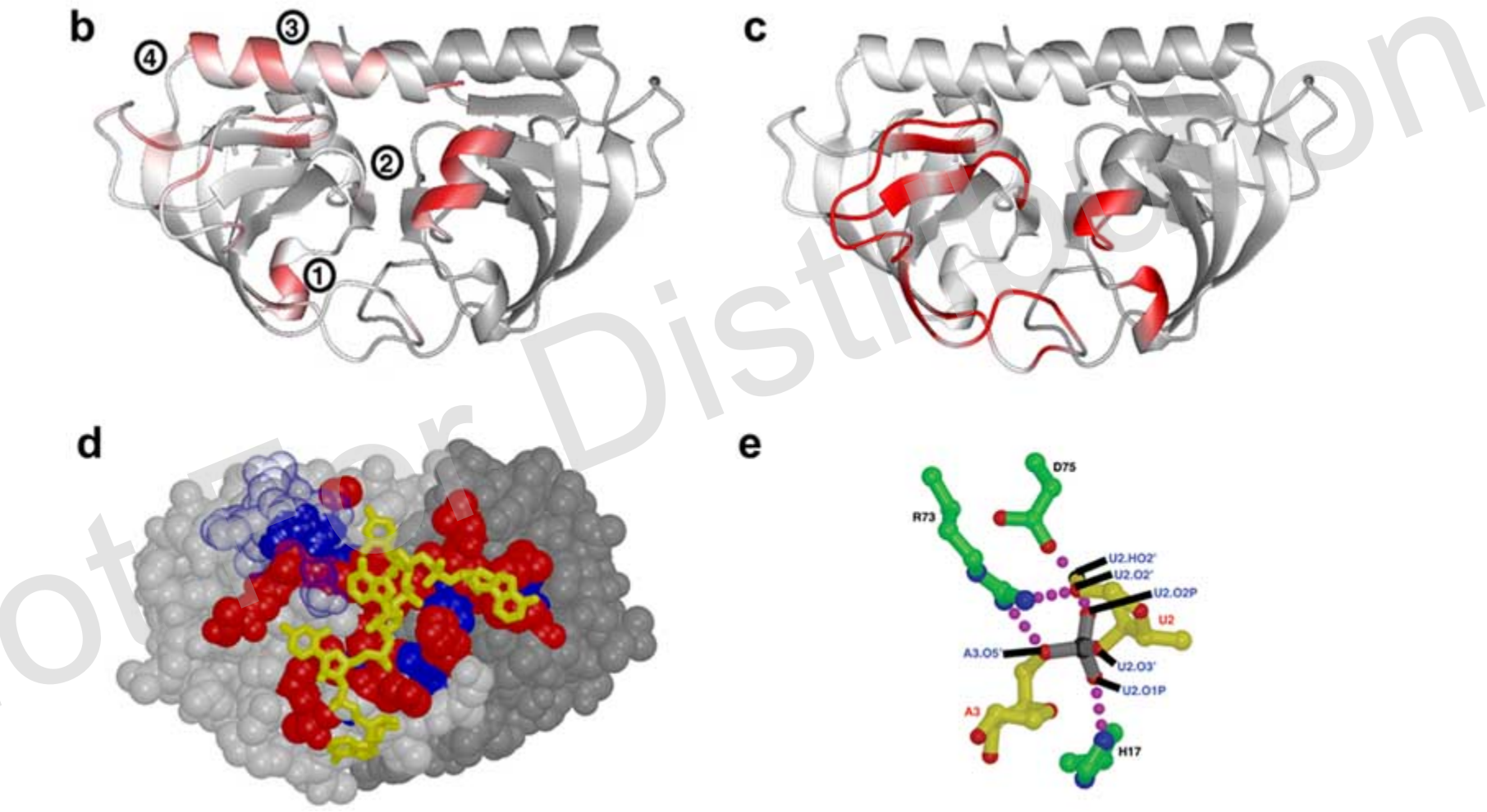

Figure 3. Overlapping antitoxin and RNA binding sites of Kid. a) Kid-Kis interactions mapped on a ribbon representations of the hexameric $\mathrm{Kid}_{2}-\mathrm{Kis}_{2}-\mathrm{Kid}_{2}$ model. The Kid-Kis hexamer is shown in two shades of grey. Kid residues affected by the addition of Kis are depicted in red, with light to dark red representing a mild to strong effect. Kid exists as a symmetric dimer and therefore two sets of originally identical residues can be distinguished. For clarity, however, only one of those sets is coloured red on each dimer. Kis residues affected by Kid binding are shown in yellow (first monomer) and blue (second monomer). The four interaction sites and the loop between $\beta$-strands 1 and 2 , comprising residues S10 to G21, are indicated. b) Kid residues affected by the addition of Kis mapped in red on a ribbon representation of the crystal structure of the Kid dimer (PDB: 1M1F). The colour coding is equal to Figure a. c) Kid residues affected by the addition of AdUACA mapped in red on a ribbon representation of the crystal structure of the Kid dimer (PDB: 1M1F). The two monomers are coloured light and dark grey. Only one set of affected residues is coloured. d) Model of the Kid-RNA complex obtained with HADDOCK (PDB: 2C06). The RNA fragment is coloured yellow, the two Kid monomers light and dark grey, and the active Kid residues red (HADDOCK definition [69]). The other Kid residues affected by the addition of AdUACA are shown in blue, with the loop in cluster three transparent to show the residues lying behind it. e) Detailed view of the catalytic site of Kid. A part of the RNA fragment is shown in yellow with the phosphate at the cleavage site in grey. Kid residues H17, R73 and D75, involved in RNA cleavage, are coloured green. Nitrogen atoms are coloured blue and oxygen atoms red. Hydrogen bonds are shown as purple dashed lines. Figures $\mathbf{a}$, b and $\mathbf{c}$ were generated with the program MOLMOL version 2K.1 [109] and Figures d and e with Molscript [110] and Raster3D [111]. Figures d and e have been reprinted from [60], with permission from Elsevier. 
a

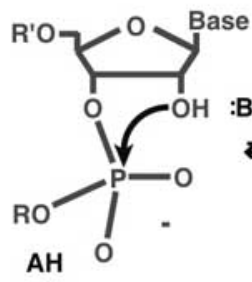

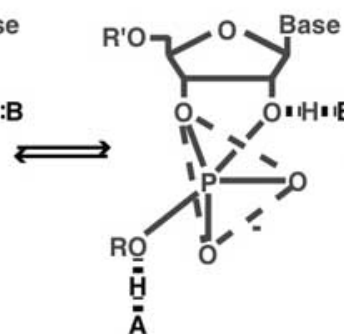

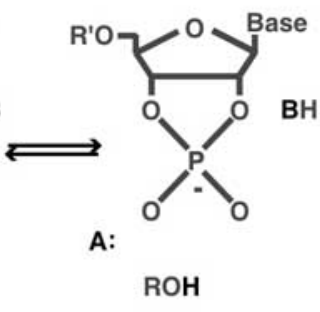

b

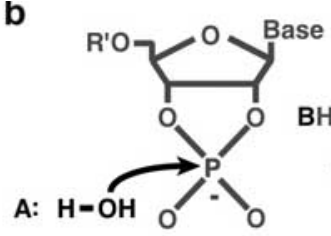

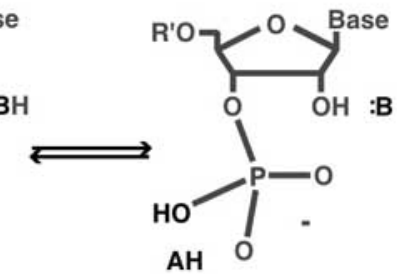

C
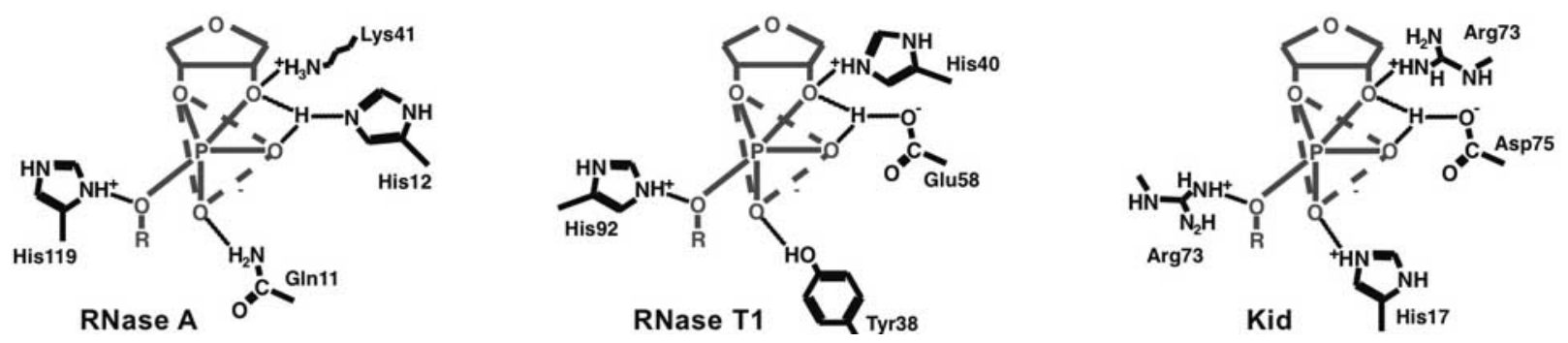

Figure 4. Catalyses by ribonucleases RNase A, RNase T1 and Kid. a) Transphosphorylation reaction of RNase A and T1 in which the catalytic base B deprotonates the 2'-OH group of the ribonucleotide that subsequently attacks the electrophilic phosphorus. A hydrogen atom of the catalytic acid A is transferred to the leaving group and via a triester-like intermediate a 2':3'-cyclic phosphate and 5'-OH group are formed. b) Hydrolysis of the 2':3'-cyclophosphate results in a 3'-monophosphate nucleotide and recycled acid and base residues. c) Schematic representation of the active site of RNase A, RNase T1 and Kid, with RNA atoms in grey and Kid atoms in black. Shown is the pentavalent transition state: the 3'-O and two nonbridging oxygens form a trigonal bipyramid (grey dashed lines), while the attacking 2'-O and leaving 5'-O take up apical positions [65]. Catalytic hydrogen bonds are depicted as black dashed lines. This Figure has been reprinted from [60], with permission from Elsevier.

map the substrate-binding face of MazF [80]. Their result closely resembles the RNA-binding site of Kid in our KidRNA complex [60]. The antitoxin MazE prevents RNA cleavage by MazF [77,79] by binding to the substratebinding site of the toxin $[43,80]$.

\section{Potentially Lethal Conditions}

Numerous conditions triggering the toxic action of MazF have been described: stressful conditions like high temperatures, oxidative stress, and DNA damage by thymine starvation, addition of mitomycin $\mathrm{C}$ or nalidixic acid, or UV irradiation $[81,82]$; the presence of protein synthesis inhibiting antibiotics such as ribfampicine, chloramphenicol and spectinomycin [83]; activation of the plasmid-borne toxin Doc (death on curing), which probably inhibits translation [81]; artificial overproduction of ppGpp that was reported to inhibit the expression of mazEF [75], although this could not be reproduced by other researchers [76]. All these situations result in the inability of the cell to replenish the antitoxin MazE upon degradation by ClpAP and thus allow MazF to express its potentially lethal activity. MazF-induced inhibition of cell growth and colony formation was shown to be reversible by overexpression of MazE up to six hours after MazF induction [14,84]. Whether mazEF functions as a bacterial system for programmed cell death [85], or rather has a bacteriostastic effect and functions in quality control of gene expression [6], still remains to be elucidated.

\section{THE CcdB-CcdA SYSTEM}

The next TA system is another well-studied plasmid encoded system, comprising the toxin $\mathrm{CcdB}(11.7 \mathrm{kD})$ and its antidote CcdA $(8.3 \mathrm{kD})$. This TA system involves the plasmid F locus $c c d$ [86,87], which stands for coupled cell division since it was originally thought to couple plasmid replication and cell division $[24,88]$. In addition to its role upon plasmid loss during cell division, it has recently been suggested that this TA system is also important for bacteria in the stationary phase, for example during nutritional stress [89].

\section{Structures and Interactions}

The crystal structure of CcdB is shown in Fig. 1 [37]. In fact, the CcdB dimer was the first toxin structure that was solved. The crystal structures of Kid and MazF resemble that of $\mathrm{CcdB}$, despite a low sequence homology between $\mathrm{CcdB}$ and the other two toxins [34]. The similarity of their secondary structure elements is shown in Fig. 2. The small threestranded antiparallel $\beta$-sheet of $\mathrm{CcdB}$, inserted in a loop of the main five-stranded antiparallel $\beta$-sheet, is thought to be the CcdA binding site [37]. The solution structure of the mutant CcdA-R70K antitoxin has been solved by NMR spectroscopy [41]. Remarkably, despite the structural similarities of the toxins, CcdA has a very different structure compared to Kis and MazE. Each monomer of the dimeric CcdA protein consists of an $\mathrm{N}$-terminal domain that contains a $\beta$-sheet followed by two $\alpha$-helices, and an unstructured $\mathrm{C}$-terminal tail. Its topology and DNA-binding properties classify the Nterminal domain as a member of the ribbon-helix-helix fold [41]. The C-terminus of the antitoxin is responsible for the binding to CcdB: a truncated form of CcdA lacking the first $31 \mathrm{~N}$-terminal residues was still able to neutralise the CcdB toxicity [90] and an NMR titration experiment showed that only CcdA residues between V46 and D71 interact with 


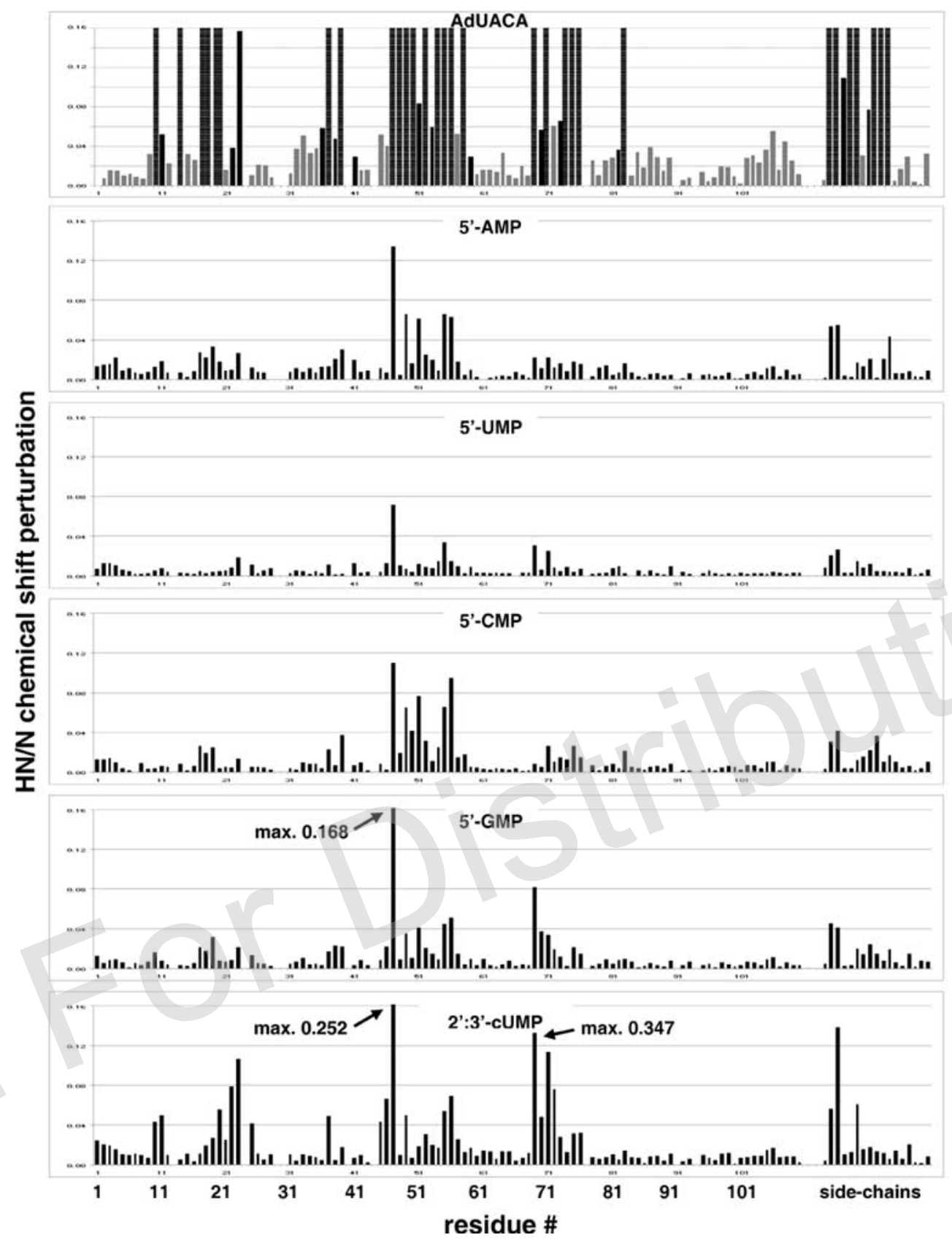

Figure 5. Chemical shift perturbations of Kid residues upon addition of AdUACA and mononucleotide ligands. The perturbations are measured at a RNA-dU:Kid ratio of 2:1 or ligand:Kid ratio of 38:1. For the RNA-dU addition, the shifts of residues in fast exchange are shown in grey and those of residues in fast to intermediate exchange in black. The dashed lines indicate disappearing residues in intermediate exchange. Side-chain NH chemical shift changes of W7/Q19-2x/Q20-2x/R23/R38/N50-2x/R73/Q76-2x/ R78/R85/N99-2x/R104 are depicted as well. T29/E91 and the side-chains of R3/N34/R35/R53/R67/R89/R92, which could not be assigned unambiguously, are not perturbed by RNA-dU or any of the mononucleotide ligands. Prolines (residues 13/24/30/40/44/77/94) can not be observed in this experiment and are therefore omitted.

CcdB [41]. Apparently, CcdA binds to CcdB in a similar way as MazE binds to MazF, via interactions of a carboxyterminal tail $[38,41]$. This is corroborated by the observation of a $\mathrm{CcdB}_{4} \mathrm{CcdA}_{2}$ heterohexamer after mixing $\mathrm{CcdB}$ and CcdA at a 2:1 ratio [91]. Like for Kid and Kis, the CcdBCcdA interaction is complex since a molar ratio of the two proteins of 1:1 resulted in a mixture of complexes with varying stoichiometry [91]. A $\left(\mathrm{CcdB}_{2} \mathrm{CcdA}_{2}\right)_{n}$ complex with a $\mathrm{CcdB}: \mathrm{CcdA}$ ratio of one binds DNA and represses the tran- scription of $c c d[51,52,54,92,93]$. When the level of $\mathrm{CcdB}$ exceeds that of CcdA, no DNA binding is observed [54]. In contrast with other TA systems, CcdA alone is not able to repress transcription [51,52,94]. The solution structure of CcdA bound to a 6 bp palindromic DNA sequence within the operator-promoter region of the $c c d$ operon has been reported [41]. The antitoxin is degraded by the ATP-dependant Lon protease [52,55]. 


\section{Mode of Action of the CcdB Toxin}

Although the structures of $\mathrm{CcdB}$, Kid and MazF are rather similar, the toxicity of $\mathrm{CcdB}$ and the other two proteins involve completely different actions. CcdB lacks numerous amino acids responsible for the RNase activity of Kid and MazF. Instead, the target of CcdB is the DNA topoisomerase gyrase [95-97]. Gyrase is an indispensable enzyme during DNA replication and transcription, since it introduces negative supercoils in circular DNA. The CcdB protein poisons gyrase by binding the GyrA dimer with its three Cterminal residues W99, G100 and I101 [37,92], causing two different effects depending on the state GyrA is present. Binding of CcdB to free GyrA or gyrase in the absence of DNA results in GyrA/gyrase-CcdB complexes unable to bind and supercoil DNA $[95,98]$. When CcdB binds gyrase during the catalytic cycle, it induces gyrase-mediated DNA cleavage resulting in a covalent gyrase-DNA complex that blocks transcription by RNA polymerase [99]. The latter process, however, is rather slow and is thought to be possible only during relocation of the T-segment DNA $[97,100]$. The most likely model for the CcdB-GyrA interaction is that CcdB binds an open conformation of the GyrA dimer. This would explain both poisoning modes, since the two catalytic tyrosine residues of gyrase are separated too far to catalyse DNA cleavage or religation [37,97]. Addition of the antitoxin CcdA releases the gyrase from the CcdB toxin [101].

\section{THE RelE-RelB SYSTEM}

The next TA system discussed here is formed by the toxin RelE $(10.3 \mathrm{kD})$ and antitoxin RelB $(8.8 \mathrm{kD})$, encoded by the relBE locus of the E. coli chromosome (K-12 strain) [33]. This system is homologous to the relBE system encoded by plasmid P307. The relBE locus is highly abundant on the chromosomes of very diverse prokaryotes: 129 loci are identified in both gram-negative and gram-positive bacteria and 27 in archaea [15,30].

\section{Structure of the aRelE-aRelB Complex}

The crystal structure of a complex of the archaeal homologues aRelE $(9.7 \mathrm{kD})$ and aRelB $(7.5 \mathrm{kD})$, expressed by the hyperthermophilic Pyrococcus horikoshii OT3, is shown in Fig. 1. The proteins form a heterotetramer (aRelE-aRelB $)_{2}$, with the four $\alpha$-helices and short $\beta$-strand of aRelB being wrapped around aRelE. The ellipsoidal aRelE consists of three $\alpha$-helices and a five-stranded $\beta$-sheet, with $\beta 2$ to $\beta 5$ running antiparallel and $\beta 1$ parallel to the neighbouring $\beta 5$. Site-directed mutagenesis has indicated that residues R40, L48, R58, R65 and especially R85 are important for the toxic activity of aRelE [40]. RelE is structurally related to the YoeB toxin and also to the microbial RNases barnase and RNase Sa [42].

\section{Mode of Action of the RelE Toxin}

RelE has been demonstrated to inhibit protein synthesis in vitro by promoting the cleavage of mRNAs in the ribosomal A-site [102]. Since aRelE has comparable dimensions and shape as the decoding domain in EF-G, the two proteins have been suggested to enter the ribosomal A-site in a similar way [40]. RelE usually promotes cutting between the second and third base; it prefers the stop codon UAG, but also cleaves the other stop codons and the sense codons UCG and CAG. Furthermore, cleavage of mRNA in the ribosomal E-site was observed after removal of the peptide from peptidyl-tRNA in the P-site, while degradation of mRNA bound to free 30S subunits was shown as well [102]. The ribonucleic acid tmRNA is thought to recover RelEinhibited protein synthesis in vivo by rescuing ribosomes stalled on mRNAs truncated by RelE [103]. RelE activity can be neutralised through the induction of transcription of the RelB gene [14].

\section{The RelE-RelB System as Stress-Response Element}

RelE toxicity is induced upon a strong inhibition of protein synthesis, as happens when the stringent response takes place. Subsequently, the existing pool of RelB antitoxin is degraded by the Lon protease and de-repression of the relBE locus follows, since the transcription is autoregulated by a cooperative action of RelB and RelE $[33,56]$. The resulting increase of relBE mRNA and the concomitant continued synthesis of RelB were suggested to allow fine-tuning of the RelB:RelE ratio [56]. It is thought that during the stringent response, due to the action of RelE, a fresh supply of amino acids is obtained via degradation of incomplete nascent proteins recognised by their tmRNA tag. Those amino acids can be used for the synthesis of proteins essential for the changed circumstances, like amino acid synthesising enzymes. An additional function of RelE could be to reduce the number of ribosomes 'consuming' the scarce amino acids in the elongation process, thereby increasing the speed and accuracy of the ribosomes that remain active. In this way, RelE would allow the cell to adjust to the new situation, resolve the nutritional stress and restore normal growth conditions. Therefore, at present, the RelE-RelB TA system is considered to be a stress-response element rather than a cell-killing module [102]. However, it was shown that in the case of the homologous pneumococcal RelBE2 system, prolonged exposure of the cells to the toxin resulted in the inability to rescue the cultivability of the cells by subsequent expression of the antitoxin [104].

\section{THE YoeB-YefM SYSTEM}

The last TA system described here comprises the toxin YoeB $(10 \mathrm{kD})$ and antitoxin YefM $(10 \mathrm{kD})[42,105,106]$. This TA system, encoded by the yefM-yoeB locus of the $E$. coli chromosome, shows similarities to the relBE system.

\section{Structures and Interactions}

Crystal structures of the YoeB-YefM complex and the free YoeB toxin have been determined [42], and the structure of the heterotrimeric YoeB-YefM $\mathrm{M}_{2}$ complex is depicted in Fig. 1. The compact, globular YoeB protein consists of a five-stranded $\beta$-sheet and two $\alpha$-helices [42]. It is highly similar to the aRelE monomer, except for an extended loop in aRelE. YoeB shows also structural similarities to RNase Sa from Streptomyces aureofaciens and Barnase from Bacillus amyloliquefaciens. The bound and free structures of YoeB are essentially identical, except for the three most Cterminal amino acids. In the free form, those residues fold into a typical conformation seen in the catalytic site of microbial RNases. The YefM homodimer can be divided in a 
globular and symmetrical N-terminal part, and two extended $\mathrm{C}$-terminal segments. The $\mathrm{N}$-terminal region consists of a single hydrophobic core of six $\beta$-strands and two $\alpha$-helices from each monomer, plus a third helix from each monomer that cross each other at an angle of $65^{\circ}$. One of the two Cterminal regions interacts with YoeB and is ordered into an $\alpha$-helix and an extended $\beta$-strand, while the other is disordered [42]. This fits well with the observation that free YefM is unfolded under native conditions [107]. YefM fragments including the $\mathrm{H} 4$ helix consisting of residues $67-83$, but not the $\mathrm{H} 4$ helix alone, where able to bind YoeB and neutralise its toxicity. Free YoeB forms a dimeric structure in which the hydrophobic interactions between the two monomers mimic the hydrophobic interactions within the YoeB-YefM complex [42].

\section{Mode of Action of the YoeB Toxin}

It was shown that the YoeB toxin induces cleavage of translated mRNAs in vivo [108] and that it functions as a ribosome-independent ribonuclease in vitro [42]. YefM is able to inhibit the RNase activity of YoeB [42], while Lon overproduction specifically activates YoeB-dependent mRNA cleavage [108]. In vivo, YoeB preferentially cuts between the second and third base (AAA, CUG, GCG, and the stop codon UAA), but also cleaves between codons (between AAA and GCU, after UAC, and after UUA) [108]. In vitro, YoeB was shown to be a purine-specific endoribonuclease [42].

\section{The Catalytic Site of YoeB}

Because of the structural similarities between YoeB and the microbial RNase $\mathrm{Sa}$ and Barnase, a similar reaction mechanism has been proposed that involves a histidine and glutamic acid residue as general acid and base, respectively, and an arginine for binding the reactive phosphate [42]. Candidates would be the YoeB residues H83, E46 and R65. In the YoeB-YefM 2 complex, the distance between the potentially catalytic groups of H83 and E46 is $~ 21 \AA$, three times larger than generally seen. In the free YoeB protein, however, where the three $\mathrm{C}$-terminal residues adopt a specific fold also observed in the catalytic site of microbial RNases, this distance is significantly smaller. Site-directed mutagenesis showed that all of those three residues, and Y84, are essential for the toxicity of YoeB. The role of Y84 would be to stabilise the protein-RNA interaction at the active site. It was concluded that YefM inhibits the catalytic activity of YoeB by stabilising non-optimal conformations of the involved residues and direct obstruction of the catalytic site [42].

\section{CONCLUDING REMARKS}

Toxin-antitoxin systems are highly conserved and abundant in plasmids and genomes of both bacteria and archaea. During normal growth conditions, the unstable antitoxin is bound to the toxin neutralising its toxicity. Upon stress, however, a diminished level of antitoxin allows the activation of the toxin causing cell growth arrest and possibly cell death. This review discusses five related, well-studied TA systems found in E. coli. A high structural similarity exists between the toxins $\mathrm{Kid}, \mathrm{MazF}$ and $\mathrm{CcdB}$, respectively the antitoxins Kis and MazE, while the structure of $\mathrm{CcdA}$ is rather different. The toxins RelE and YoeB, respectively the antitoxins RelB and YefM, are also structurally related, but their complexes possess different stoichiometries.

CcdB causes gyrase poisoning in contrast to the other four toxins that are RNases. RelE functions by cleaving mRNAs in the ribosomal A-site, while Kid, MazF and YoeB seem more effective in ribosome-independent RNA cleavage. A model for RNA binding and the catalytic site of Kid has been determined and it was suggested that D75 and R73 are the catalytic base and acid, respectively. Sequence alignments show that D75 of Kid is conserved in MazF (D76) and most related toxins (D or E), but that R73 is not. For speculation about the catalytic acid of MazF and other related toxins, the structures of these proteins in their active conformation are needed. At the position corresponding to $\mathrm{H} 17$, the third active site residue of Kid, a histidine or functionally similar serine is found. A significant overlap exists between the RNA and antitoxin binding sites of the two toxins Kid and MazF. This explains the mode of inhibition by Kis and MazE, respectively, on a molecular level. The CcdB-CcdA and RelE-RelB complexes might be too large to enter their specific site of action. The catalytic residues of YoeB have been proposed to be E46 and H83, being the catalytic base and acid, respectively. R65 is thought to bind the reactive phosphate. The observed absence of catalytic residues in RelE and their presence in YoeB is consistent with the intrinsic ribonuclease activity of YoeB and the fact that RelE mediates cleavage of mRNA in a ribosomedependent manner.

For the structurally related TA systems, i.e. Kid-Kis, MazF-MazE and CcdB-CcdA, the obtained information seems to point out a general 'architecture' of the systems. Likely, those and related TA systems all function according to a same scheme, following here. In a bacterial cell experiencing a normal growth situation, the antitoxin:toxin ratio is approximately $4: 1$. The complexes formed at these conditions are multimers with antitoxin:toxin ratios close to $1: 1$; the toxicity is neutralised and transcription of the TA operon is autoregulated properly. Possibly, the 1:1 stoichiometry of the TA multimers is favourable for protein-DNA interactions. Upon loss of the plasmid or other stressful conditions, antitoxin synthesis is abolished and the antitoxin:toxin ratio drops significantly; $\mathrm{T}_{2} \mathrm{~A}_{2} \mathrm{~T}_{2}$ heterohexamers are formed to neutralise the toxicity as much as possible. For plasmidencoded systems, a progressive activation of the toxin will occur in time. Gradually, toxin dimers will co-exist with the heterohexamers and affect cell growth increasingly. The same might happen for chromosomal TA systems. However, since de-repression of the operon follows upon diminishing of the antitoxin, a continued synthesis of antitoxin might take place. This could result in survival of the cell.

\section{ACKNOWLEDGEMENTS}

We would like to thank G.E. Folkers for valuable discussion. This project was financially supported by the European Union (QLK2-CT-2000-00634, HPRI-CT-2001-00172 and NMR Large Scale Facility scheme). M.B. Kamphuis was further supported by the Center for Biomedical Genetics, M.C. Monti by a Short-Term FEBS Fellowship and the 
lab of R. Díaz-Orejas by the Spanish MCyT (SAF200204649) and MEC (BFU2005-03911/BMC).

\section{REFERENCES}

[1] Hayes, F. (2003) Science, 301, 1496-1499.

[2] Holcik, M. and Iyer, V. N. (1997) Microbiology, 143 ( Pt 11), 3403-3416.

[3] Engelberg-Kulka, H. and Glaser, G. (1999) Annu. Rev. Microbiol., 53, 43-70.

[4] Gerdes, K., Rasmussen, P. B. and Molin, S. (1986) Proc. Natl. Acad. Sci. USA, 83, 3116-3120.

[5] Jensen, R. B. and Gerdes, K. (1995) Mol. Microbiol., 17, 205-210.

[6] Gerdes, K., Christensen, S. K. and Lobner-Olesen, A. (2005) Nat. Rev. Microbiol., 3, 371-382.

[7] Greenfield, T. J., Ehli, E., Kirshenmann, T., Franch, T., Gerdes, K. and Weaver, K. E. (2000) Mol. Microbiol., 37, 652-660.

[8] Condon, C. (2006) Mol. Microbiol., 61, 573-583.

[9] Anantharaman, V. and Aravind, L. (2003) Genome Biol., 4, R81.

[10] Engelberg-Kulka, H., Sat, B., Reches, M., Amitai, S. and Hazan, R. (2004) Trends Microbiol., 12, 66-71.

[11] Hayes, C. S. and Sauer, R. T. (2003) Cell, 112, 2-4

[12] Nystrom, T. (2003) Bioessays, 25, 204-211.

[13] Nystrom, T. (2004) Annu. Rev. Microbiol., 58, 161-181.

[14] Pedersen, K., Christensen, S. K. and Gerdes, K. (2002) Mol. Microbiol., 45, 501-510.

[15] Pandey, D. P. and Gerdes, K. (2005) Nucleic Acids Res., 33, 966976.

[16] Gerdes, K. (2000) J. Bacteriol., 182, 561-572.

[17] Clissold, P. M. and Ponting, C. P. (2000) Curr. Biol., 10, R888890.

[18] de la Cueva-Mendez, G., Mills, A. D., Clay-Farrace, L., DiazOrejas, R. and Laskey, R. A. (2003) EMBO J., 22, 246-251.

[19] Picardeau, M., Le Dantec, C., Richard, G. F. and Saint Girons, I. (2003) FEMS Microbiol. Lett., 229, 277-281.

[20] Yamamoto, T. A., Gerdes, K. and Tunnacliffe, A. (2002) FEBS Lett., 519, 191-194.

[21] Gabant, P., Van Reeth, T., Dreze, P. L., Faelen, M., Szpirer, C. and Szpirer, J. (2000) Biotechniques, 28, 784-788.

[22] Xu, W., Zhang, Y., Yeh, L. Y., Ruprecht, C. R., Wong-Staal, F., McFadden, B. A., Reddy, T. R. and Ruprecht, R. M. (2002) Biotechniques, 32, 1266-8, 1270.

[23] Bernard, P., Gabant, P., Bahassi, E. M. and Couturier, M. (1994) Gene, 148, 71-4.

[24] Ogura, T. and Hiraga, S. (1983) Proc. Natl. Acad. Sci. USA, 80, 4784-4788.

[25] Bravo, A., de Torrontegui, G. and Diaz, R. (1987) Mol. Gen. Genet., 210, 101-110.

[26] Pullinger, G. D. and Lax, A. J. (1992) Mol. Microbiol., 6, 16311643.

[27] Lehnherr, H., Maguin, E., Jafri, S. and Yarmolinsky, M. B. (1993) J. Mol. Biol., 233, 414-428.

[28] Roberts, R. C. and Helinski, D. R. (1992) J. Bacteriol., 174, 81198132.

[29] Tian, Q. B., Ohnishi, M., Tabuchi, A. and Terawaki, Y. (1996) Biochem. Biophys. Res. Commun., 220, 280-284.

[30] Gronlund, H. and Gerdes, K. (1999) J. Mol. Biol., 285, 1401-1415.

[31] Metzger, S., Dror, I. B., Aizenman, E., Schreiber, G., Toone, M., Friesen, J. D., Cashel, M. and Glaser, G. (1988) J. Biol. Chem., 263, 15699-15704.

[32] Masuda, Y., Miyakawa, K., Nishimura, Y. and Ohtsubo, E. (1993) J. Bacteriol., 175, 6850-6856.

[33] Gotfredsen, M. and Gerdes, K. (1998) Mol. Microbiol., 29, 10651076.

[34] Hargreaves, D., Santos-Sierra, S., Giraldo, R., Sabariegos-Jareno, R., de la Cueva-Mendez, G., Boelens, R., Diaz-Orejas, R. and Rafferty, J. B. (2002) Structure (Camb), 10, 1425-1433.

[35] Ceglowski, P., Boitsov, A., Chai, S. and Alonso, J. C. (1993) Gene, 136, 1-12.

[36] Brantl, S., Behnke, D. and Alonso, J. C. (1990) Nucleic Acids Res., $18,4783-4790$.
[37] Loris, R., Dao-Thi, M. H., Bahassi, E. M., Van Melderen, L., Poortmans, F., Liddington, R., Couturier, M. and Wyns, L. (1999) J. Mol. Biol., 285, 1667-1677.

[38] Kamada, K., Hanaoka, F. and Burley, S. K. (2003) Mol. Cell, 11, 875-884.

[39] Loris, R., Marianovsky, I., Lah, J., Laeremans, T., EngelbergKulka, H., Glaser, G., Muyldermans, S. and Wyns, L. (2003) $J$. Biol. Chem., 278, 28252-28257.

[40] Takagi, H., Kakuta, Y., Okada, T., Yao, M., Tanaka, I. and Kimura, M. (2005) Nat. Struct. Mol. Biol., 12, 327-331.

[41] Madl, T., Van Melderen, L., Mine, N., Respondek, M., Oberer, M., Keller, W., Kathai, L. and Zangger, K. (2006) J. Mol. Biol., online.

[42] Kamada, K. and Hanaoka, F. (2005) Mol. Cell, 19, 497-509.

[43] Kamphuis, M. B. et al. (2007) Proteins, on-line, doi: 10,1002/ prot.21254.

[44] Tsuchimoto, S., Ohtsubo, H. and Ohtsubo, E. (1988) J. Bacteriol., 170, 1461-1466.

[45] Bravo, A., Ortega, S., de Torrontegui, G. and Diaz, R. (1988) Mol. Gen. Genet., 215, 146-151.

[46] Lemonnier, M., Santos-Sierra, S., Pardo-Abarrio, C. and DiazOrejas, R. (2004) J. Bacteriol., 186, 240-243.

[47] Ruiz-Echevarria, M. J., Berzal-Herranz, A., Gerdes, K. and DiazOrejas, R. (1991) Mol. Microbiol., 5, 2685-2693.

[48] Santos-Sierra, S., Pardo-Abarrio, C., Giraldo, R. and Diaz-Orejas, R. (2002) FEMS Microbiol. Lett., 206, 115-119.

[49] Tsuchimoto, S. and Ohtsubo, E. (1993) Mol. Gen. Genet., 237, 8188 .

[50] Marianovsky, I., Aizenman, E., Engelberg-Kulka, H. and Glaser, G. (2001) J. Biol. Chem., 276, 5975-5984

[51] Tam, J. E. and Kline, B. C. (1989) Mol. Gen. Genet., 219, 26-32.

[52] Salmon, M. A., Van Melderen, L., Bernard, P. and Couturier, M. (1994) Mol. Gen. Genet., 244, 530-538.

[53] Zhang, J., Zhang, Y. and Inouye, M. (2003) J. Biol. Chem., 278, 32300-32306.

[54] Afif, H., Allali, N., Couturier, M. and Van Melderen, L. (2001) Mol. Microbiol., 41, 73-82.

[55] Van Melderen, L., Thi, M. H., Lecchi, P., Gottesman, S., Couturier M. and Maurizi, M. R. (1996) J. Biol. Chem., 271, 27730-27738.

[56] Christensen, S. K., Mikkelsen, M., Pedersen, K. and Gerdes, K (2001) Proc. Natl. Acad. Sci. USA, 98, 14328-14333.

[57] Tsuchimoto, S., Nishimura, Y. and Ohtsubo, E. (1992) J. Bacteriol., 174, 4205-4211.

[58] Hargreaves, D., Giraldo, R., Santos-Sierra, S., Boelens, R., Rice, D. W., Diaz Orejas, R. and Rafferty, J. B. (2002) Acta Crystallogr. D Biol. Crystallogr., 58, 355-358.

[59] Santos-Sierra, S., Lemonnier, M., Nunez, B., Hargreaves, D., Rafferty, J., Giraldo, R., Andreu, J. M. and Diaz-Orejas, R. (2003) Plasmid, 50, 120-130.

[60] Kamphuis, M. B., Bonvin, A. M. J. J., Monti, M. C., Lemonnier, M., Muñoz-Gómez, A., van den Heuvel, R. H. H., Díaz-Orejas, R. and Boelens, R. (2006) J. Mol. Biol., 357, 115-126.

[61] Munoz-Gomez, A. J., Lemonnier, M., Santos-Sierra, S., BerzalHerranz, A. and Diaz-Orejas, R. (2005) J. Bacteriol., 187, 31513157.

[62] Zhang, J., Zhang, Y., Zhu, L., Suzuki, M. and Inouye, M. (2004) $J$. Biol. Chem., 279, 20678-20684.

[63] Pimentel, B., Madine, M. A. and de la Cueva-Mendez, G. (2005) Embo J., 24, 3459-3469.

[64] Loverix, S. and Steyaert, J. (2001) Methods Enzymol., 341, 305 323.

[65] Loverix, S. and Steyaert, J. (2003) Curr. Med. Chem., 10, 779-785.

[66] Raines, R. T. (1998) Chem. Rev., 98, 1045-1066.

[67] Steyaert, J. (1997) Eur. J. Biochem., 247, 1-11.

[68] Ruiz-Echevarria, M. J., de la Torre, M. A. and Diaz-Orejas, R (1995) FEMS Microbiol. Lett., 130, 129-135.

[69] Dominguez, C., Boelens, R. and Bonvin, A. M. (2003) J. Am Chem. Soc., 125, 1731-1737.

[70] Kamphuis, M. B. (2005) Utrecht University, Utrecht.

[71] Gogos, A., Mu, H., Bahna, F., Gomez, C. A. and Shapiro, L. (2003) Proteins, 53, 320-322.

[72] Pellegrini, O., Mathy, N., Gogos, A., Shapiro, L. and Condon, C. (2005) Mol. Microbiol., 56, 1139-1148. 
[73] Lemos, J. A., Brown, T. A., Jr., Abranches, J. and Burne, R. A. (2005) FEMS Microbiol. Lett., 253, 251-257.

[74] Zhu, L., Zhang, Y., Teh, J. S., Zhang, J., Connell, N., Rubin, H. and Inouye, M. (2006) J. Biol. Chem., 281, 18638-18643.

[75] Aizenman, E., Engelberg-Kulka, H. and Glaser, G. (1996) Proc. Natl. Acad. Sci. USA, 93, 6059-6063.

[76] Christensen, S. K., Pedersen, K., Hansen, F. G. and Gerdes, K. (2003) J. Mol. Biol., 332, 809-819.

[77] Zhang, Y., Zhang, J., Hoeflich, K. P., Ikura, M., Qing, G. and Inouye, M. (2003) Mol. Cell, 12, 913-923.

[78] Zhang, Y., Zhang, J., Hara, H., Kato, I. and Inouye, M. (2005) J. Biol. Chem., 280, 3143-3150.

[79] Munoz-Gomez, A. J., Santos-Sierra, S., Berzal-Herranz, A., Lemonnier, M. and Diaz-Orejas, R. (2004) FEBS Lett., 567, 316320.

[80] Li, G. Y., Zhang, Y., Chan, M. C., Mal, T. K., Hoeflich, K. P., Inouye, M. and Ikura, M. (2006) J. Mol. Biol., 357, 139-150.

[81] Hazan, R., Sat, B. and Engelberg-Kulka, H. (2004) J. Bacteriol., 186, 3663-3669.

[82] Sat, B., Reches, M. and Engelberg-Kulka, H. (2003) J. Bacteriol., 185, 1803-1807.

[83] Sat, B., Hazan, R., Fisher, T., Khaner, H., Glaser, G. and Engelberg-Kulka, H. (2001) J. Bacteriol., 183, 2041-2045.

[84] Amitai, S., Yassin, Y. and Engelberg-Kulka, H. (2004) J. Bacteriol., 186, 8295-8300.

[85] Kolodkin-Gal, I. and Engelberg-Kulka, H. (2006) J. Bacteriol. $188,3420-3423$

[86] Hiraga, S., Jaffe, A., Ogura, T., Mori, H. and Takahashi, H. (1986) J. Bacteriol., 166, 100-104.

[87] Jaffe, A., Ogura, T. and Hiraga, S. (1985) J. Bacteriol., 163, 841849.

[88] Miki, T., Yoshioka, K. and Horiuchi, T. (1984) J. Mol. Biol., 174, 605-625.

[89] Aguirre-Ramírez, M., Ramírez-Santos, J., Van Melderen, L. and Gómez-Eichelmann, M. C. (2006) Canad. J. Microbiol., 52, 24-30.

[90] Bernard, P. and Couturier, M. (1991) Mol. Gen. Genet., 226, $297-$ 304.
[91] Dao-Thi, M. H., Charlier, D., Loris, R., Maes, D., Messens, J., Wyns, L. and Backmann, J. (2002) J. Biol. Chem., 277, 3733-3742.

[92] Bahassi, E. M., Salmon, M. A., Van Melderen, L., Bernard, P. and Couturier, M. (1995) Mol. Microbiol., 15, 1031-1037.

[93] de Feyter, R., Wallace, C. and Lane, D. (1989) Mol. Gen. Genet., 218, 481-486.

[94] Tam, J. E. and Kline, B. C. (1989) J. Bacteriol., 171, 2353-2360.

[95] Maki, S., Takiguchi, S., Miki, T. and Horiuchi, T. (1992) J. Biol. Chem., 267, 12244-12251.

[96] Bernard, P. and Couturier, M. (1992) J. Mol. Biol., 226, 735-745.

[97] Dao-Thi, M. H., Van Melderen, L., De Genst, E., Afif, H., Buts, L., Wyns, L. and Loris, R. (2005) J. Mol. Biol., 348, 1091-1102.

[98] Maki, S., Takiguchi, S., Horiuchi, T., Sekimizu, K. and Miki, T. (1996) J. Mol. Biol., 256, 473-482.

[99] Critchlow, S. E., O'Dea, M. H., Howells, A. J., Couturier, M., Gellert, M. and Maxwell, A. (1997) J. Mol. Biol., 273, 826-839.

[100] Kampranis, S. C., Howells, A. J. and Maxwell, A. (1999) J. Mol. Biol., 293, 733-744.

[101] Bahassi, E. M., O'Dea, M. H., Allali, N., Messens, J., Gellert, M. and Couturier, M. (1999) J. Biol. Chem., 274, 10936-10944.

[102] Pedersen, K., Zavialov, A. V., Pavlov, M. Y., Elf, J., Gerdes, K. and Ehrenberg, M. (2003) Cell, 112, 131-140.

[103] Christensen, S. K. and Gerdes, K. (2003) Mol. Microbiol., 48 , 1389-1400.

[104] Nieto, C., Pellicer, T., Balsa, D., Christensen, S. K., Gerdes, K. and Espinosa, M. (2006) Mol. Microbiol., 59, 1280-1296.

[105] Cherny, I., Rockah, L. and Gazit, E. (2005) J. Biol. Chem., 280, 30063-30072.

[106] Grady, R. and Hayes, F. (2003) Mol. Microbiol., 47, 1419-1432.

[107] Cherny, I. and Gazit, E. (2004) J. Biol. Chem., 279, 8252-8261.

[108] Christensen, S. K., Maenhaut-Michel, G., Mine, N., Gottesman, S., Gerdes, K. and Van Melderen, L. (2004) Mol. Microbiol., 51, 1705-1717.

[109] Koradi, R., Billeter, M. and Wuthrich, K. (1996) J. Mol. Graph., $14,51-5,29-32$

[110] Kraulis, P. J. (1991) J. Appl. Crystallogr., 24, 946-950.

[111] Merritt, E. A. and Murphy, M. E. (1994) Acta Crystallogr. D Biol. Crystallogr., 50, 869-873. 